\title{
Pharmacological interactions between intrathecal pregabalin plus tianeptine or clopidogrel in a rat model of neuropathic pain
}

\author{
Hyung Gon Lee ${ }^{1,2, *}$, Yeo Ok Kim ${ }^{1, *}$, Jeong II Choi ${ }^{1,2}$, Xue Hao Han ${ }^{1,2}$, Yang Un Shin ${ }^{1}$, and Myung Ha Yoon ${ }^{1,2}$ \\ 'Department of Anesthesiology and Pain Medicine, Chonnam National University Medical School, Gwangju, Korea \\ ${ }^{2}$ BioMedical Sciences Graduate Program (BMSGP), Chonnam National University, Gwangju, Korea
}

Received October 15, 2021

Revised November 29, 2021

Accepted November 29, 2021

Handling Editor: Jong Yeon Park

\section{Correspondence}

Myung Ha Yoon

Department of Anesthesiology and Pain Medicine, Chonnam National University

Medical School, 42, Jebong-ro, Dong-gu,

Gwangju 61469, Korea

Tel: +82-62-220-6895

Fax: +82-62-232-6294

E-mail: mhyoon@jnu.ac.kr

*These authors contributed equally to this work as the first author.
Background: There is still unmet need in treating neuropathic pain and increasing awareness regarding the use of drug combinations to increase the effectiveness of treatment and reduce adverse effects in patients with neuropathic pain.

Methods: This study was performed to determine the individual and combined effects of pregabalin, tianeptine, and clopidogrel in a rat model of neuropathic pain. The model was created by ligation of the L5-L6 spinal nerve in male Sprague-Dawley rats; mechanical allodynia was confirmed using von Frey filaments. Drugs were administered to the intrathecal space and mechanical allodynia was assessed; drug interactions were estimated by isobolographic or fixed-dose analyses.

Results: Intrathecal pregabalin and tianeptine increased the mechanical withdrawal threshold in a dose-dependent manner, but intrathecal clopidogrel had little effect on the mechanical withdrawal threshold. An additive effect was noted between pregabalin and tianeptine, but not between pregabalin and clopidogrel.

Conclusions: These findings suggest that intrathecal coadministration of pregabalin and tianeptine effectively attenuated mechanical allodynia in the rat model of neuropathic pain. Thus, pregabalin plus tianeptine may be a valid option to enhance the efficacy of neuropathic pain treatment.

Key Words: Chronic Pain; Clopidogrel; Drug Interactions; Hyperalgesia; Neuralgia; Pregabalin; Rats; Spinal Nerves; Tianeptine.

\section{INTRODUCTION}

Neuropathic pain is defined by the International Association for the Study of Pain as "pain caused by a lesion or disease of the somatosensory nervous system" [1]. It has been linked to a poor quality of life in $7 \%-8 \%$ of adults. Nevertheless, many patients do not receive proper treatment for neuropathic pain [2], due to its diverse mechanisms, the difficulty of diagnosis, ineffective treatments, and side effects associated with those treatments [3]. Although combination therapy has an inconclusive GRADE (the Grading of Recommendations Assessment, Development, and Evaluation) classification [3], combined treatment with anticonvulsants and antidepressant drugs (both primary drugs for neuropathic pain) may be an option for physicians expecting to see synergistic effects; this approach leads to enhanced efficacy and reduced side effects. In addition, to enable appropriate drug selection based on neuropathic pain pathophysiology, there is substantial interest in identification of novel targets.

Pregabalin, an anticonvulsant that acts on calcium channels, results in the inhibition of neurotransmitter (c) This is an open-access article distributed under the terms of the Creative Commons Attribution Non-Commercial License (http://creativecommons.org/licenses/by-nc/4.0/), which permits unrestricted non-commercial use, distribution, and reproduction in any medium, provided the original work is properly cited.

(c) The Korean Pain Society, 2022
Author contributions: Hyung Gon Lee: Writing/manuscript preparation; Yeo Ok Kim: Writing/manuscript preparation; Jeong II Choi: Data curation; Xue Hao Han: Investigation; Yang Un Shin: Investigation; Myung Ha Yoon: Supervision. 
release; it has demonstrated efficacy in postherpetic neuralgia, diabetic peripheral neuropathy, and post-traumatic neuropathic pain [4]. Tianeptine, an antidepressant with distinct neurochemical properties, selectively enhances serotonin reuptake; however, there have been few published studies regarding its analgesic effects [5]. Recently, P2Y12 receptor antagonists have garnered attention due to their relationships with pathological pain (e.g., neuropathic or inflammatory pain) [6,7]. Clopidogrel, a P2Y12 receptor antagonist, is broadly used as an antiplatelet agent in clinical situations. This study was performed to determine the individual and combined effects of pregabalin, tianeptine, and clopidogrel in a rat model of neuropathic pain.

\section{MATERIALS AND METHODS}

\section{Animal preparation}

In these experiments, Male Sprague-Dawley rats (weighing 150-180 g) were used; rats were maintained on a $12 \mathrm{hr}$ light/dark cycle at a well-controlled temperature of $24^{\circ} \mathrm{C}$. Food and water were provided ad libitum. The Institutional Animal Care and Use Committee, Chonnam National University approved the experimental protocol (CNU IACUC-H-2014-11).

\section{Neuropathic pain animal model}

The spinal nerve ligation method was used to induce neuropathic pain [8]. With rats under sevoflurane anesthesia, the left L5 and L6 spinal nerves near the vertebral column were separated and tightly ligated using $6-0$ silk. Rats were considered to exhibit neuropathy when they demonstrated mechanical allodynia (i.e., paw flinching behavior in response to the application of a bending force of $<4 \mathrm{~g}$ ). If motor impairment was induced after the procedure, rats were euthanized by an overdose of sevoflurane.

\section{Intrathecal catheterization}

At 5 days after spinal nerve ligation, with the rats under sevoflurane anesthesia, a polyethylene tube (PE-10; Becton Dickinson Co., Sparks, MD) was implanted into the intrathecal space through an incision made in the atlantooccipital membrane to the level of the lumbar enlargement [9]. After catheter implantation, rats were provided a recovery period of 7 days; they were closely monitored and excluded if they exhibited neurologic deficits.

\section{Drugs}

The drugs used were pregabalin (Pfizer Inc., New York, NY), tianeptine (JEIL Pharm. Co., Ltd., Seoul, Korea), and clopidogrel hydrogen sulfate (Tocris Bioscience, Bristol, UK); all drugs were dissolved in saline. Using a handdriven, gear-operated syringe pump, drugs were injected as a solution in a volume of $0.01 \mathrm{~mL}$ intrathecally; $0.01 \mathrm{~mL}$ additional normal saline was flushed into the catheter.

\section{Assessment of mechanical allodynia}

Rats were acclimatized for 30 minutes in a separate transparent container with a wire mesh floor and the test was conducted with calibrated von Frey filaments from underneath the cage to the hind paw. Eight von Frey filaments $(0.4,0.7,1.2,2.0,3.6,5.5,8.5$, and $15 \mathrm{~g})$ were sequentially applied vertically to the plantar surface of the hind paw for 5 seconds, while the hair was bent. After mechanical stimulation with von Frey filaments, quick paw avoidance was considered a positive result. In accordance with the up and down method, lighter filaments were used after a positive reaction and heavier filaments were used after a negative reaction [10]. After the first positive reaction, a series of five or six experiments was conducted. The lightest filament that exhibited a withdrawal reaction was defined as the paw withdrawal threshold. The absence of a positive response to the pressure of the $15 \mathrm{~g}$ von Frey filament was regarded as the cut-off value.

\section{Experimental paradigm}

On the day of the experiment (7 days after intrathecal cannulation), rats that had undergone an adaptation time of 30 minutes were allocated into two groups: experimental and control. Test drugs were randomly delivered. In the control group, intrathecal saline was delivered at an identical volume to that of the experimental group. Rats were only used in the experiment once, and the investigator was blinded to the drug used. The effects of intrathecal tianeptine $(30,100,300 \mu \mathrm{g})$, clopidogrel $(100,300,500 \mu \mathrm{g})$, and pregabalin $(0.3,1,3 \mu \mathrm{g})$ on neuropathic pain were evaluated (6-7 rats per group). Baseline mechanical withdrawal thresholds were checked prior to drug injection. At 15, 30, $60,90,120,150$, and 180 minutes after intrathecal drug injection, the paw withdrawal threshold was evaluated. The maximum drug dose was determined based on a pilot study without neurological deficit. The antinociceptive effect of the drugs was evaluated as the percentage of the maximal possible effect (\% MPE). 
\% MPE $=([$ post drug threshold - post injured baseline threshold] / [cutoff threshold - post injured baseline threshold] $) \times 100$.

\section{Drug interaction}

Isobolographic and fixed-dose analyses were performed to assess drug interactions between pregabalin and tianeptine or clopidogrel [11]. Isobolographic analysis determines the equipotent dose of drugs. From the dose-response curves of two drugs (pregabalin and tianeptine), 50\% effective dose $\left(\mathrm{ED}_{50}\right)$ values were estimated, which represented the result of reducing the MPE by $50 \%$. Subsequently, by a constant dose ratio based on the $\mathrm{ED}_{50}$ of individual drugs, the dose response curve for combined administration of the two drugs was obtained. Thus, tianeptine $\mathrm{ED}_{50}$ + pregabalin $\mathrm{ED}_{50}$, (tianeptine $\mathrm{ED}_{50}+$ pregabalin $\left.\mathrm{ED}_{50}\right) / 2$, (tianeptine $\mathrm{ED}_{50}+$ pregabalin $\mathrm{ED}_{50}$ )/4, and (tianeptine $\mathrm{ED}_{50}$ + pregabalin $\left.\mathrm{ED}_{50}\right) / 8$ were injected. Thereafter, the $\mathrm{ED}_{50}$ values of the mixtures were calculated. Isobolograms were expressed by displaying the $\mathrm{ED}_{50}$ values of each drug on the $\mathrm{x}$ and $\mathrm{y}$ axes, respectively; the line connecting the two points represents the theoretical line of additivity. Furthermore, to describe the magnitude of the interaction, a "total fraction value" was used. Total fraction value $=\left(\mathrm{ED}_{50}\right.$ of drug 1 combined with drug 2) / $\left(\mathrm{ED}_{50}\right.$ for drug 1 given alone $)+\left(\mathrm{ED}_{50}\right.$ of drug 2 combined with drug 1$) /\left(\mathrm{ED}_{50}\right.$ for drug 2 given alone). The fractional values indicate what portion of the single $\mathrm{ED}_{50}$ value was accounted for by the corresponding $\mathrm{ED}_{50}$ value for the combination. Values near 1 indicate an additive interaction, values greater than 1 imply an antagonistic interaction, and values less than 1 indicate a synergistic interaction. Fixed-dose analysis was used to examine the correlation between pregabalin and clopidogrel, because clopidogrel alone had no antiallodynic effect. A fixed clopidogrel dose $(300 \mu \mathrm{g})$ was intrathecally injected with various pregabalin doses.

\section{General behavior}

Additional experiments with other rats were performed to evaluate changes in behavior at the highest dose of each drug: pregabalin $(3 \mu \mathrm{g})$, tianeptine $(300 \mu \mathrm{g})$, and clopidogrel $(500 \mu \mathrm{g})$. Placing-stepping and righting reflexes were assessed for motor functions; corneal and pinna reflexes were induced by string stimulation of the cornea or ear canal. Behavioral changes were noted as present or absent [12].

\section{Statistical analysis}

All data are expressed as the mean \pm standard error of the mean. Dose-response results were analyzed by one-way analysis of variance with Bonferroni comparison for post hoc analysis. The statistical analysis was performed using SPSS 25.0 software (IBM Corp., Armonk, NY). Doseresponse lines were fitted by least-squares linear regression; $\mathrm{ED}_{50}$ and its $95 \%$ confidence intervals were calculated in accordance with the method described by Tallarida [13]. The difference between theoretical and experimental $\mathrm{ED}_{50}$ values was examined by using $t$-tests. $P<0.05$ was considered statistically significant.

\section{RESULTS}

Tactile allodynia was induced after ligation of the L5L6 spinal nerves in experimental rats; paw withdrawal threshold was $<4 \mathrm{~g}$. No behavioral changes were observed even at the highest dose of each drug (i.e., $3 \mu \mathrm{g}$ for pregabalin, $300 \mu \mathrm{g}$ for tianeptine, and $500 \mu \mathrm{g}$ for clopidogrel) (Fig. 1). Pregabalin, tianeptine, and a mixture of pregabalin and tianeptine injected intrathecally all increased the mechanical withdrawal threshold in a dose-dependent manner (Fig. 2).

The 1:1 ratio of pregabalin and tianeptine showed additive effects in isobolographic analysis (Fig. 3). Although the experimental $\mathrm{ED}_{50}$ values were lower than the theoretical $\mathrm{ED}_{50}$ values (Fig. 3), the difference was not significant. And the total fraction value was close to 1 , indicating an additive interaction (Table 1).

Intrathecal clopidogrel had little effect on the mechanical withdrawal threshold (Fig. 2). In addition, intrathecal coadministration of a fixed clopidogrel dose $(300 \mu \mathrm{g})$ with pregabalin $(0.3,1,3 \mu \mathrm{g})$ did not affect the paw withdrawal threshold of pregabalin alone (Fig. 4).

\section{DISCUSSION}

Traditionally, approaches for management of neuropathic pain comprise conservative pharmacological and complementary treatments with a focus on symptoms. However, limited drug effectiveness, an aging patient population, multi-drug instructions, and opioid-related adverse effects reduce patient compliance [14]. Clinical studies thus far do not provide sufficient guidance for physicians; thus, further experimental studies are needed to overcome these problems. There is increasing awareness regarding the use of drug combinations to reduce adverse effects in patients with neuropathic pain. The combined use of drugs is presumably more effective and safer than monotherapy. Synergistic effects are demonstrated in terms of actions that are greater than those expected from individual potencies 


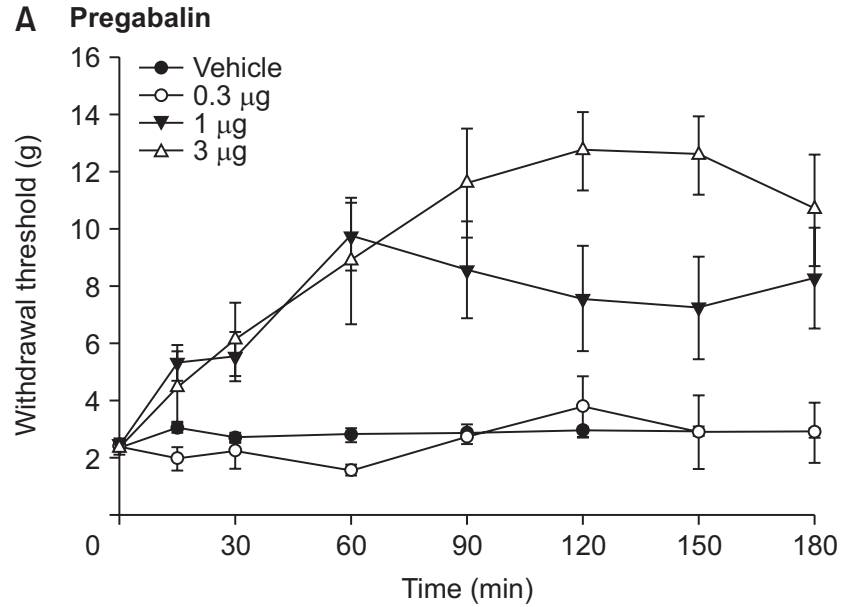

C Clopidogrel
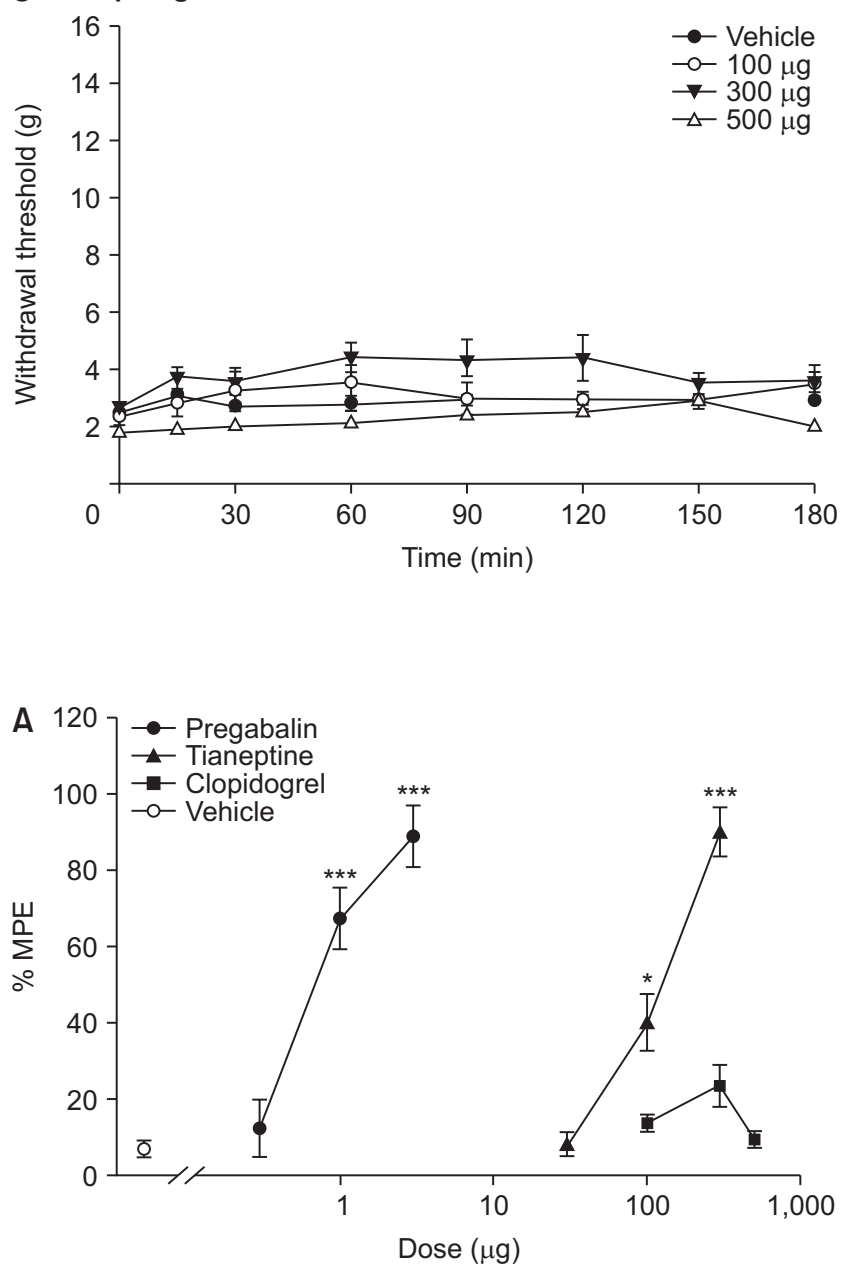

\section{B Tianeptine}

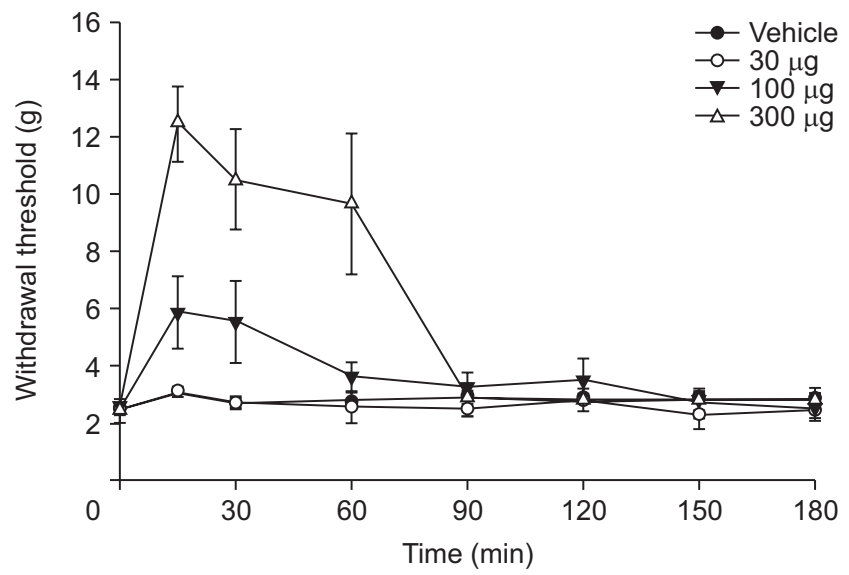

Fig. 1. Time course curves of intrathecal pregabalin (A), tianeptine (B) and clopidogrel $(\mathrm{C})$ on the hind paw withdrawal response after spinal nerve ligation. Data are presented as the withdrawal threshold (g). Each line represents the means \pm standard error of the mean of 6 rats.

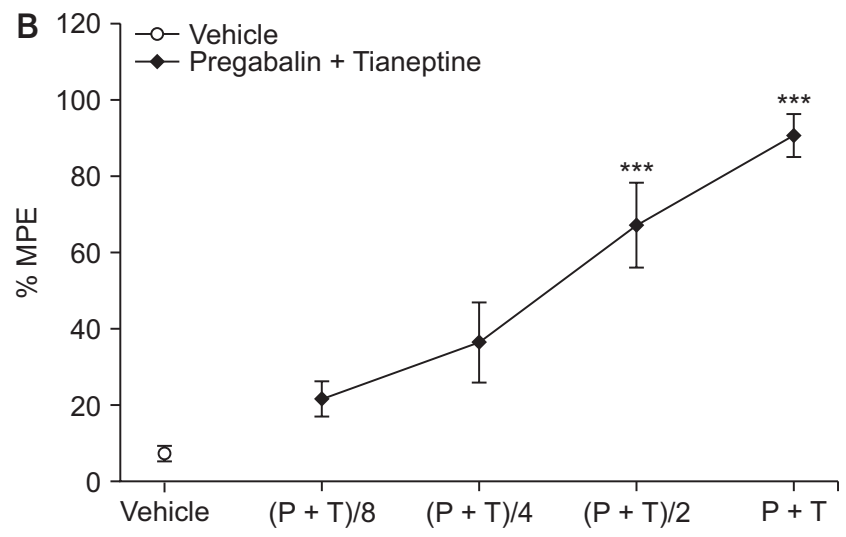

Fig. 2. Dose response effect of intrathecal pregabalin, tianeptine and clopidogrel (A), or mixture of pregabalin and tianeptine (B) on the hind paw withdrawal response after spinal nerve ligation. Data are presented as the percent of maximal possible effect (\% MPE). Each line represents the means \pm standard error of the mean of 6 rats. $\mathrm{P}$ : pregabalin $\mathrm{ED}_{50}$, T: tianeptine $\mathrm{ED}_{50}, \mathrm{ED}_{50}: 50 \%$ effective dose. $* P<0.05, * * * P<0.001$, compared with vehicle.

and efficacies [15].

In the present study, pregabalin and tianeptine relieved tactile allodynia, whereas clopidogrel had minimal effect on allodynia. These results indicate that both pregabalin and tianeptine can be effectively used to manage spinal nerve ligation-induced neuropathy, whereas clopidogrel cannot. Pregabalin is a well-known anticonvulsant and analgesic agent that is a first-line drug for neuropathic pain. In preclinical and clinical settings, the effectiveness of pregabalin is well established $[16,17]$. Various animal 


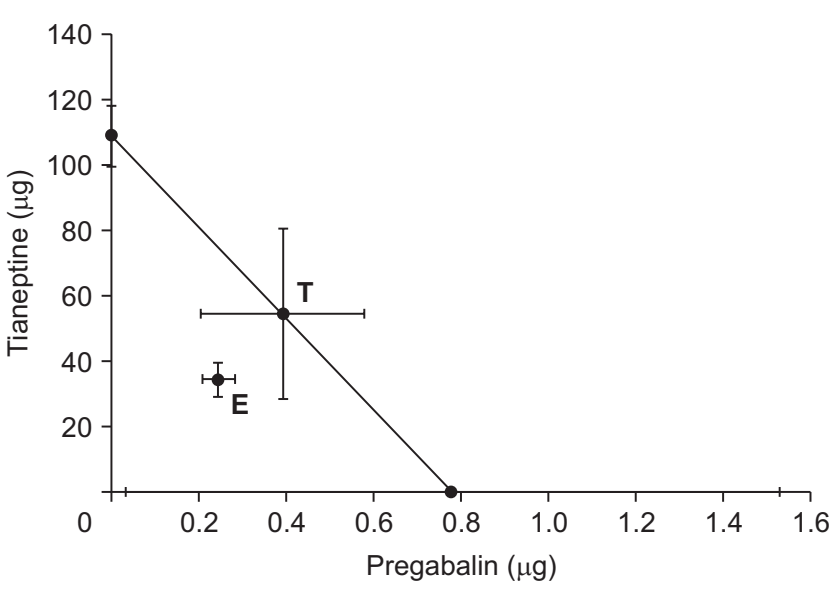

Fig. 3. Isobologram for the interaction between intrathecal pregabalin and tianeptine. The $\mathrm{ED}_{50}$ values for each agent are plotted on the $\mathrm{x}$ axis and $y$ axis, respectively. The experimental $\mathrm{ED}_{50}$ point $(\mathrm{E})$ was significantly different from the theoretical $E D_{50}$ point $(T)$, indicating an additive interaction in pregabalin and tianeptine. $\mathrm{ED}_{50}: 50 \%$ effective dose.

Table 1. $\mathrm{ED}_{50}$ values ( $\left.\mu \mathrm{g}\right)$ with $95 \% \mathrm{Cl}$ and $\mathrm{TFV}$ of intrathecal drugs

\begin{tabular}{lcc}
\hline \multicolumn{1}{c}{ Drug } & $\mathrm{ED}_{50}(95 \% \mathrm{Cl})$ & $\mathrm{TFV}$ \\
\hline Pregabalin & $0.78(0.56-1.10)$ & - \\
Tianeptine & $108.80(87.46-135.35)$ & - \\
Tianeptine $^{\mathrm{a}}$ :Pregabalin & $34.33(26.83-43.92)$ & 0.63 \\
Pregabalin $^{\mathrm{a}}$ :Tianeptine & $0.24(0.19-0.31)$ & - \\
\hline
\end{tabular}

$\mathrm{ED}_{50}$ : effective dose producing a $50 \%$ reduction of control response, $\mathrm{Cl}$ : confidence intervals, TFV: total fraction value, -: not available.

${ }^{a}$ These values imply the $\mathrm{ED}_{50}$ values of tianeptine and pregabalin in the mixture of tianeptine and pregabalin.

models of neuropathic and inflammatory pain, including spinal nerve ligation, have revealed the effectiveness of pregabalin [18]. Its effectiveness has also been proven in treatment of clinical conditions such as diabetic neuropathy, chemotherapy-induced peripheral neuropathy, postherpetic neuralgia, fibromyalgia, and trigeminal neuralgia [18]. Similar to gabapentin, the action site of pregabalin is the $\alpha 2 \delta$-subunit of voltage-gated calcium channels in the central nervous system [19]. Blockage of this site can reduce depolarization-induced calcium influx and neurotransmitter release. Reduced levels of neurotransmitters (e.g., glutamate, norepinephrine, and substance P) decrease abnormal neuronal excitability, which appears to promote antinociceptive effects [20].

Tianeptine has demonstrated antiallodynic effects on neuropathic and inflammatory pain. It has superior tolerability, compared to conventional antidepressant medications, because it exhibits fewer side effects (i.e., sedation, cardiovascular changes, cognitive changes, sexual dysfunction, and nausea) [21]. Preclinical studies showed that the administration of tianeptine enhanced extracellular levels of 5-HT and norepinephrine in the spinal

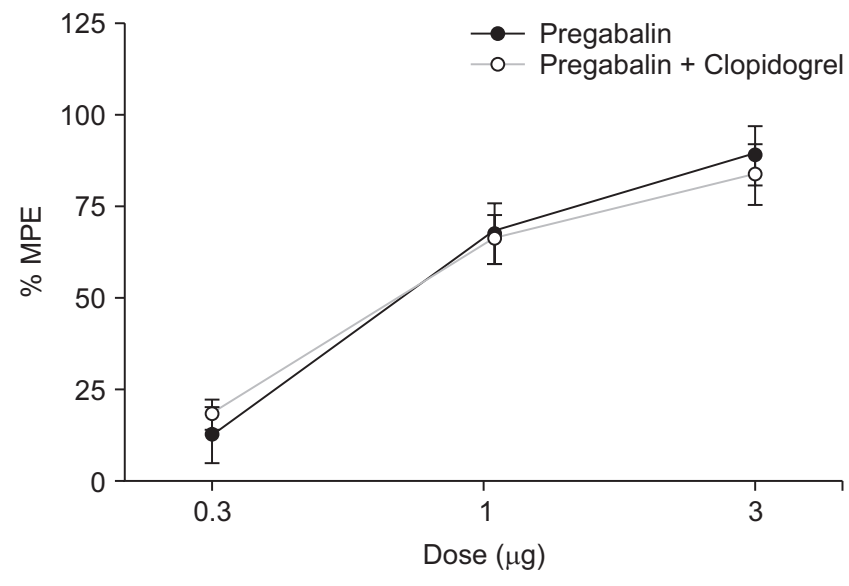

Fig. 4. A fixed dose analysis for the interaction between intrathecal clopidogrel $(300 \mu \mathrm{g})$ and pregabalin. Data are presented as the percent of maximal possible effect (\% MPE). Each line represents the means \pm standard error of the mean of 6 rats.

dorsal horn [22], enhanced 5-HT uptake in the brain, and reduced stress-induced atrophy of neuronal dendrites [23]. Therefore, serotonergic and noradrenergic transmission may contribute to the antinociceptive effect of tianeptine. Recently, an opioid-related mechanism was proposed to explain the antinociceptive effects of tianeptine. Antagonists of all opioid receptor types $(\mu, \delta$, and $\kappa)$ prevented the antinociceptive effect of tianeptine in rats with visceral nociception [24].

Clopidogrel, a P2Y12 receptor antagonist, is broadly used as an antiplatelet agent in clinical situations related to cardiovascular conditions. P2Y12 is a P2Y metabotropic G-protein-coupled purinergic receptor that is a new target of neuropathic pain therapy. Previous studies demonstrated that specific antagonists of P2Y12 receptors (e.g., clopidogrel) alleviated tactile allodynia in rodent models of neuropathic pain [6,7]. However, this study failed to confirm the effect of P2Y12 receptor antagonist.

Considering the use of multi-drug treatment in clinical practice, the possibility of combination agents is an important issue. In the present study, pregabalin interacted with tianeptine in an additive manner, whereas it did not interact with clopidogrel. These findings suggest that a mixture of pregabalin plus tianeptine can provide drug combination benefits, while a mixture of clopidogrel plus pregabalin may be ineffective for neuropathic pain management.

Although the endpoint of pharmacological interactions between two types of agents is complicated, the agents may interact with each other by altering the kinetics at target receptors or channels [19]. Thus, if agents with different or shared mechanisms are coadministered, a synergistic or an additive interaction is expected. Previous studies demonstrated synergistic or additive interaction between 
some drugs $[25,26]$. It has been reported that the antinociceptive effects of gabapentin and tianeptine were reversed by an opioid receptor antagonist at the spinal level $[24,27]$. On the other hand, serotonergic and noradrenergic systems are involved in the antinociception of tianeptine, but not that of gabapentin $[24,27]$. These findings suggest that both drugs may have not only a common pharmacologic site of action but also different mechanisms of action. In the present study, the experimental $\mathrm{ED}_{50}$ values were not significantly different from the theoretical $\mathrm{ED}_{50}$ values. Furthermore, the total fraction value was close to 1 . Thus, it could be assumed that pregabalin interacted with tianeptine additively rather than synergistically.

The absence of reinforced interaction between pregabalin and clopidogrel in the present study may be due to the ineffectiveness of clopidogrel itself on neuropathic pain. Notably, the antiallodynic effect of pregabalin was observed at a late stage, while tianeptine showed an antiallodynic effect at an early stage. These phenomena may have led to complementary effects following treatment with pregabalin plus tianeptine.

In conclusion, intrathecal injections of pregabalin plus tianeptine may be useful for management of neuropathic pain. The enhanced efficacy of this combination will aid physicians in clinical treatment. Further investigations are needed regarding the dose of each drug to be used in the clinical setting.

\section{CONFLICT OF INTEREST}

No potential conflict of interest relevant to this article was reported.

\section{FUNDING}

This study was supported by a grant (BCRI18027-1) of Chonnam National University Hospital Biomedical Research Institute.

\section{ORCID}

Hyung Gon Lee, https://orcid.org/0000-0003-4898-4355 Yeo Ok Kim, https://orcid.org/0000-0002-1748-8079 Jeong Il Choi, https://orcid.org/0000-0002-3571-2599 Xue Hao Han, https://orcid.org/0000-0002-6369-6792 Yang Un Shin, https://orcid.org/0000-0001-9652-6637 Myung Ha Yoon, https://orcid.org/0000-0002-9962-0255

\section{REFERENCES}

1. IASP. Taxonomy: neuropathic pain [Internet]. Washington, D.C.: IASP; 2017. Available at: https://www.iasp-pain.org/ resources/terminology/?ItemNumber=1698\#Neuropathicpa in.

2. IASP. Epidermiology of neuropathic pain: how common is neuropathic pain, and what is its impact? [Internet]. Washington, D.C.: IASP; 2014. Available at: https:// www.iasp-pain.org/advocacy/global-year/neuropathicpain/?itemNumber $=3934$.

3. Finnerup NB, Attal N, Haroutounian S, McNicol E, Baron R, Dworkin RH, et al. Pharmacotherapy for neuropathic pain in adults: a systematic review and meta-analysis. Lancet Neurol 2015; 14: 162-73.

4. Derry S, Bell RF, Straube S, Wiffen PJ, Aldington D, Moore RA. Pregabalin for neuropathic pain in adults. Cochrane Database Syst Rev 2019; 1: CD007076.

5. Heo BH, Shin JY, Park KS, Lee HG, Choi JI, Yoon MH, et al. Effects of tianeptine on the development and maintenance of mechanical allodynia in a rat model of neuropathic pain. Neurosci Lett 2016; 633: 82-6.

6. Tozaki-Saitoh H, Tsuda M, Miyata H, Ueda K, Kohsaka S, Inoue K. P2Y12 receptors in spinal microglia are required for neuropathic pain after peripheral nerve injury. J Neurosci 2008; 28: 4949-56.

7. Yu T, Zhang X, Shi H, Tian J, Sun L, Hu X, et al. P2Y12 regulates microglia activation and excitatory synaptic transmission in spinal lamina II neurons during neuropathic pain in rodents. Cell Death Dis 2019; 10: 165.

8. Kim SH, Chung JM. An experimental model for peripheral neuropathy produced by segmental spinal nerve ligation in the rat. Pain 1992; 50: 355-63.

9. Yaksh TL, Rudy TA. Chronic catheterization of the spinal subarachnoid space. Physiol Behav 1976; 17: 1031-6.

10. Chaplan SR, Bach FW, Pogrel JW, Chung JM, Yaksh TL. Quantitative assessment of tactile allodynia in the rat paw. J Neurosci Methods 1994; 53: 55-63.

11. Yoon MH, Choi JI, Kwak SH. Characteristic of interactions between intrathecal gabapentin and either clonidine or neostigmine in the formalin test. Anesth Analg 2004 ;98: 1374-9.

12. Yoon MH, Choi JI, Jeong SW. Antinociception of intrathecal cholinesterase inhibitors and cholinergic receptors in rats. Acta Anaesthesiol Scand 2003; 47: 1079-84.

13. Tallarida RJ. Drug synergism and dose-effect data analysis. Boca Raton, Chapman and Hall/CRC. 2000.

14. Colloca L, Ludman T, Bouhassira D, Baron R, Dickenson AH, Yarnitsky D, et al. Neuropathic pain. Nat Rev Dis Primers 2017; 3: 17002.

15. Tallarida RJ. Quantitative methods for assessing drug synergism. Genes Cancer 2011; 2: 1003-8.

16. Sałat K, Librowski T, Nawiesniak B, Gluch-Lutwin M. Evalu- 
ation of analgesic, antioxidant, cytotoxic and metabolic effects of pregabalin for the use in neuropathic pain. Neurol Res 2013; 35: 948-58.

17. Zhang SS, Wu Z, Zhang LC, Zhang Z, Chen RP, Huang YH, et al. Efficacy and safety of pregabalin for treating painful diabetic peripheral neuropathy: a meta-analysis. Acta Anaesthesiol Scand 2015; 59: 147-59.

18. Verma V, Singh N, Singh Jaggi A. Pregabalin in neuropathic pain: evidences and possible mechanisms. Curr Neuropharmacol 2014; 12: 44-56.

19. Belliotti TR, Capiris T, Ekhato IV, Kinsora JJ, Field MJ, Heffner TG, et al. Structure-activity relationships of pregabalin and analogues that target the alpha(2)-delta protein. J Med Chem 2005; 48: 2294-307.

20. Ben-Menachem E. Pregabalin pharmacology and its relevance to clinical practice. Epilepsia 2004; 45 Suppl 6: 13-8.

21. Sindrup SH, Otto M, Finnerup NB, Jensen TS. Antidepressants in the treatment of neuropathic pain. Basic Clin Pharmacol Toxicol 2005; 96: 399-409.

22. Lee HG, Choi JI, Yoon MH, Obata H, Saito S, Kim WM. The antiallodynic effect of intrathecal tianeptine is exerted by increased serotonin and norepinephrine in the spinal dorsal horn. Neurosci Lett 2014; 583: 103-7.

23. Uzbay TI. Tianeptine: potential influences on neuroplasticity and novel pharmacological effects. Prog Neuropsychopharmacol Biol Psychiatry 2008; 32: 915-24.

24. Bilge SS, İlkaya F, Darakcı Ö, Çiftcioğlu E, Bozkurt A. Opioid receptors contribute to antinociceptive effect of tianeptine on colorectal distension-induced visceral pain in rats. Pharmacology 2018; 101: 96-103.

25. Jung YH, Kim YO, Han JH, Kim YC, Yoon MH. Isobolographic analysis of drug combinations with intrathecal BRL52537 ( -opioid agonist), pregabalin (calcium channel modulator), AF 353 (P2X3 receptor antagonist), and A804598 (P2X7 receptor antagonist) in neuropathic rats. Anesth Analg 2017; 125: 670-7.

26. Yoon MH, Bae HB, Choi JI, Jeong SW, Chung SS, Yoo KY, et al. Evaluation of interaction between intrathecal adenosine and MK801 or NBQX in a rat formalin pain model. Pharmacology 2005; 75: 157-64.

27. Yoon MH, Choi JI, Jeong SW. Spinal gabapentin and antinociception: mechanisms of action. J Korean Med Sci 2003; 18: 255-61. 